\title{
Association between mild stimulated IVF/M cycle and early embryo arrest in sub fertile women with/without PCOS
}

\author{
Nagwa Elshewy ${ }^{1}$, Dongmei ji1,2,3 , Zhiguo Zhang ${ }^{1,3,4}$, Dawei Chen ${ }^{1,3,4}$, Beili Chen ${ }^{1,2,3}$, Rufeng Xue ${ }^{1,2,3}$, Huan Wu $\mathbf{W u}^{1,3}$,
} Jianye Wang ${ }^{1,3}$, Ping Zhou ${ }^{1,2,3,4,5^{*}}$ and Yunxia Cao ${ }^{1,2,3,4,5^{*}}$

\begin{abstract}
Background: The in vitro maturation (IVM) technique has physical and financial benefits, but a lower efficiency and outcome that is still unclear whether it is related to polycystic ovary syndrome (PCOS) itself or the IVM procedure. In this study, we analyzed the clinical and laboratory outcomes of an optimized IVM protocol in patients with and without PCOS. We also discussed the possible reasons for early embryo arrest in the IVM cycle.

Methods: This prospective study involved 58 PCOS patients and 56 non-PCOS patients who underwent mild stimulated IVF combined IVM (IVF/M) cycles. The clinical and laboratory outcomes were compared between the two groups. Also, metaphase II (MII) oocytes were obtained after IVM from the two groups, and in vivo MII oocytes randomly collected from IVF patients were examined for mitochondrial function using a laser scanning confocal microscope (LSCM). The aneuploidy rate for arrested cleavage embryos from IVM and IVF oocytes were screened using Next Generation Sequencing (NGS).

Results: Mildly stimulated IVF/M resulted in cumulative clinical pregnancy and implantation rates $(40.2,28.7 \%$ in the PCOS group vs. $41.9,36 \%$ in the non-PCOS group), respectively. The blastocyst formation rates were comparable (28\% vs. $28.2 \%$ ) in PCOS and non-PCOS groups, respectively. Using LSCM, there was a significant decrease in the mitochondrial membrane potential of IVM oocytes compared with the control IVF oocytes $(P<0.001)$, but no significant difference between the PCOS and non-PCOS groups. The NGS showed that the aneuploidy rates were comparable $(75,75$, and 66.6\%) in IVM-PCOS, IVM-non-PCOS, and control IVF arrested embryos, respectively.

Conclusions: The mildly stimulated IVF/M protocol produced acceptable clinical outcomes in PCOS and non-PCOS patients. IVM itself rather than the PCOS condition adversely affected the embryo development through its effect on mitochondrial function, which appeared to be a possible cause for the embryo arrest in the IVM cycles rather than chromosomal aneuploidy.
\end{abstract}

Keywords: IVM, Embryo arrest, Mitochondria, Aneuploidy

\footnotetext{
*Correspondence: zhoup_325@aliyun.com; caoyunxia6@126.com

${ }^{1}$ Reproductive Medicine Center, Department of Obstetrics and Gynecology, the First Affiliated Hospital of Anhui Medical University, Hefei, China

Full list of author information is available at the end of the article
}

(C) The Author(s). 2020 Open Access This article is licensed under a Creative Commons Attribution 4.0 International License, which permits use, sharing, adaptation, distribution and reproduction in any medium or format, as long as you give appropriate credit to the original author(s) and the source, provide a link to the Creative Commons licence, and indicate if changes were made. The images or other third party material in this article are included in the article's Creative Commons licence, unless indicated otherwise in a credit line to the material. If material is not included in the article's Creative Commons licence and your intended use is not permitted by statutory regulation or exceeds the permitted use, you will need to obtain permission directly from the copyright holder. To view a copy of this licence, visit http://creativecommons.org/licenses/by/4.0/. The Creative Commons Public Domain Dedication waiver (http://creativecommons.org/publicdomain/zero/1.0/) applies to the data made available in this article, unless otherwise stated in a credit line to the data. 


\section{Introduction}

In vitro maturation of human oocytes (IVM) is a promising laboratory technique in which germinal vesicle (GV) and metaphase I (MI) oocytes proceed to metaphase II (MII) oocytes. Both immature and mature oocytes can be retrieved in natural cycles or mildly stimulated cycles using hCG or GnRH agonist priming. Therefore, the procedure is better defined as the IVF cycle combined with IVM [1].

While a conventional belief exists that the growth of a dominant follicle suppresses the growth of the remaining smaller follicles that leads to their atresia, natural cycle IVF combined with IVM (IVF/M) has shown that these enclosed, arrested oocytes can resume meiosis and complete maturation in the lab. Natural cycle IVF/M has achieved higher cumulative clinical pregnancy rates with in-vivo mature oocytes obtained from dominant follicles and immature oocytes retrieved from smaller follicles after IVM culture [2]. The Lim-Chian protocol for natural cycle IVF/M has shown that injection of 10 , 000 IU hCG could avoid premature ovulation while the dominant follicle was at a suitable size (diameter between 12 and $14 \mathrm{~mm}$ ) [3]. Natural cycle IVF/M has been an attractive choice for infertility treatment for various reasons. However, it resulted in fewer numbers of retrieved oocytes due to small size in addition to the in vitro maturation culture defect.

Therefore, researchers have implemented various protocols to improve the outcome of IVM, including unstimulated, FSH priming, hCG priming, and FSH combined hCG priming. However, as described above, most IVM protocols used FSH priming to promote follicular growth, while the use of hCG triggering was controversial. Several embryological studies reported that the use of $10,000 \mathrm{IU}$ hCG injection before oocyte retrieval during an IVM cycle could hasten oocyte maturation in vitro and improve the maturation and developmental competence of oocytes [4-8]. However, others believed that the retrieval of mature oocytes with hCG triggered in IVM cycles was contrary to the basis of that procedure $[9,10]$. In addition, there was no definitive evidence for the effect of hCG triggering on clinical pregnancy or miscarriage rates, and live birth rate in IVM cycles [11].

Despite the advanced improvements in clinical IVM protocols that have occurred and the fact that the IVM culture media resulted in satisfactory clinical pregnancy rates comparable with standard IVF protocols in the well-selected patients [9, 12-14], IVM cycles showed a lower blastocyst formation rate compared to standard IVF cycles [15]. Furthermore, the biological loss of oocytes during in vitro maturation culture resulted in lower chances of pregnancy for some patients.
In comparison to IVF, which can achieve $40-70 \%$ blastocysts and others that arrest at different earlier stages [16-18], blastocyst embryo formation is observed to be only $20 \%$ from IVM cycles [12]. This lower percentage reflects the higher incidence of early embryo arrest in IVM cycles. Previous researchers attributed the early embryo arrest to multiple factors, including parental genetic causes, oxidative stress [19], energy stores defects [20], and aneuploidy [21].

Despite the fact that IVM is mainly indicated for patients with polycystic ovary syndrome (PCOS), it remains unclear whether the low efficiency is due to the IVM procedure or PCOS of a combination of the two factors. In the current study, we compared the clinical and laboratory outcomes of mildly stimulated IVF combined with IVM (IVF/M) in PCOS and non-PCOS patients. Furthermore, we evaluated the efficiency of the IVM technique with respect to the in vitro maturation rate, fertilization rate, cleavage embryo rate, and blastocyst formation rate for both groups.

To unravel the underlying mechanism of early embryo arrest, we used laser scanning confocal microscope (LSCM) to measure the impact of IVM culture on mitochondrial membrane potential $(\Delta \Psi \mathrm{m})$, which forms the basis of energy metabolism in cells, in MII oocytes matured in vitro in PCOS and non-PCOS groups compared to randomly collected in vivo MII oocytes. In addition, we used Next-Generation Sequencing (NGS) to determine the aneuploidy rates in arrested (IVM and IVF) embryos.

\section{Materials and methods \\ Patients}

This prospective study included 114 patients who were examined for mildly stimulated IVF/M cycles from September 2018 to November 2019 at the reproductive medical center, in the first affiliated Hospital of Anhui Medical University, Hefei, Anhui Province, China. The patients, aged <35years old, had normal basal FSH levels $(<10 \mathrm{mIU} / \mathrm{mL})$ and a body mass index (BMI) range of $19-25 \mathrm{~kg} / \mathrm{m} 2$. Patients with normal ovulatory or anovulatory cycles with any cause for infertility, including tubal factors, mild to moderate male factors, and unexplained infertility, were enrolled in this study. Patients were divided into two treatment groups. Group A included anovulatory patients diagnosed as PCOS according to Rotterdam criteria [22], and Group B included ovulatory non-PCOS patients.

On day 3 of their menstrual cycle, blood samples were obtained for endocrine tests for FSH, LH, prolactin, testosterone, and estradiol. All patients were assessed for metabolic syndrome by measuring total cholesterol, HDL, LDL cholesterol, triglycerides, and their level of 
insulin-resistance using fasting insulin and fasting glucose tests.

Patients who donated MII oocytes during COH/IVF cycles were chosen randomly to serve as a control group in the investigation of $\Delta \Psi \mathrm{m}$ under confocal microscope. These patients, aged < 35 years old $(29 \pm 2.5)$, had an average duration of infertility of $2( \pm 1.5)$ years, BMI of $22( \pm 1.6) \mathrm{kg} / \mathrm{m} 2$, FSH level of $6( \pm 1.2) \mathrm{mIU} / \mathrm{mL}$ and a LH level of $4( \pm 1.6) \mathrm{mIU} / \mathrm{mL}$. Nine MII oocytes were obtained from eight patients. Of these, two patients were diagnosed with PCOS, four patients with tubal factor infertility and two patients with unexplained infertility and normal ovarian function.

\section{Mildly stimulated IVF/M protocol}

The treatment cycle was initiated based on an ultrasound scan on day 3 of a spontaneous or a progestininduced withdrawal bleeding. All participants had a total of 7 or more antral follicles in each ovary. A short course of gonadotropins with an initial dose of $150 \mathrm{IU}$ of r-FSH (Gonal-F, Merck co, Switzerland) was administered for 4-5 days, beginning on cycle Day 3 or 4 , when the follicle size was 5 or less $\mathrm{mm}$ in diameter. The follicle diameter and endometrium thickness were assessed. Oocyte retrieval was scheduled when the follicle reached $10-12 \mathrm{~mm}$ in diameter when an hCG injection of 10, $000 \mathrm{IU}$ (Ovidrel, Merck-Serono, U.K) was applied $36 \mathrm{~h}$ before oocyte retrieval.

\section{Oocyte collection, IVM, sperm preparation, and ICSI}

Oocyte retrieval was performed using transvaginal ultrasound-guided aspiration with a 17-gauge single-lumen needle (Cook, Eight Mile Plains, Australia), connected to a portable pump with a pressure of less than $90 \mathrm{mmHg}$. Oocytes were examined on the day of aspiration for maturity after denudation of cumulus cells. The mature oocytes were inseminated using intracytoplasmic sperm injection (ICSI) on the day of oocyte retrieval. The immature oocytes were cultured for $24-48 \mathrm{~h}$ in IVM medium prepared in the IVF lab of our reproductive medical center. The IVM medium consisted of $80 \%$ IVM maturation medium, TCM-199 supplemented with 20\% of the patient's own serum, $0.05 \mathrm{IU} / \mathrm{ml} \mathrm{r}-\mathrm{FSH}, 0.05 \mathrm{IU} /$ $\mathrm{mL}$ HCG, $0.04 \mathrm{mg} / \mathrm{ml} 17$ B-estradiol, $0.2 \mathrm{mM} / \mathrm{L}$ pyruvic acid, and $0.05 \mathrm{ml}$ of melatonin. The oocytes were cultured at $37^{\circ} \mathrm{C}$ in $6 \% \mathrm{CO} 2,5 \% \mathrm{O} 2$, and $89 \% \mathrm{~N} 2$ with high humidity to allow for maturation.

The IVM culture medium consisted of a basic culture media, exogenous hormonal additives, and a source of protein. In particular, the maternal serum contains valuable components, including growth factors, amino acids, and some hormones that are essential for oocyte maturation. Moreover, these endogenous hormones can help promote oocyte maturation. In addition, PCOS patients (with a non-hyperandrogenic phenotype) recruited to our study had comparable endogenous hormonal levels with non-PCOS patients and no significant differences between the two groups of patients. Therefore, the addition of different sera did not interfere with the results of the in vitro culture of immature oocytes for the two groups.

After $24 \mathrm{~h}$, we checked for the presence of a polar body in IVM oocytes. ICSI was performed for the invitro matured oocytes, and any immature oocytes remained in IVM media for another $24 \mathrm{~h}$. Fresh sperm preparations were obtained twice on the day of ICSI for in vivo, and in vitro mature oocytes and ICSI was performed as a common routine in the IVF lab.

\section{Endometrial preparation and embryo transfer}

On the day of oocyte collection, $4 \mathrm{mg}$ estradiol valerate (Progynova, Jenapharm Gmbh, Germany) was administered twice daily for endometrial preparation for patients undergoing fresh embryo transfer (ET) with an endometrium thickness $8 \mathrm{~mm}$ or greater. Sixty $\mathrm{mg}$ progesterone in oil (Zhejiang Xianju Pharmaceuticals, china) was injected daily for luteal support starting on the day of ICSI. Two good quality cleavage embryos (more than 8 uniform cells and less than $20 \%$ fragmentation) were transferred on day 3 after insemination of the in-vitro matured oocytes. The embryos from the in-vivo matured oocytes and the remaining embryos from the in vitro matured oocytes were cultured to the blastocyst stage until day 5 or 6 , and then the good-quality embryos were cryopreserved. Two weeks after ET, the serum $\beta$-hCG level was tested to detect pregnancy. Two weeks later, clinical pregnancy was confirmed by the appearance of a gestational sac with a fetal pole using ultrasound.

\section{Analysis of oocytes' mitochondria under LSCM}

A laser scanning confocal microscope (LSCM) was used to detect the impact of IVM culture on the mitochondrial membrane potential $(\Delta \Psi \mathrm{m})$. Three groups of oocytes were tested as follows: Group A, 7 MII oocytes after IVM from 5 PCOS patients, Group B, 7 MII oocytes after IVM from 6 non-PCOS patients, and Group C, (control) 9 MII oocytes randomly collected from 8 IVF patients less than 35 years of age. The MII oocytes examined in the IVM groups were matured in vitro after $24 \mathrm{~h}$ from GV oocytes.

All oocytes in the three groups showed normal morphology and were stained using the $\Delta \Psi \mathrm{m}$ specific probe JC-1 (beyotime, C-2005), which was diluted to a final concentration of $5 \mu \mathrm{g} / \mathrm{ml}$ in embryo culture medium (G-1 +5\%HSA, Vitro life). After washing the oocytes twice with PBS, we added $0.5 \mathrm{~mL}$ of the staining solution to the oocytes and incubated them in a humidified incubator containing $6 \% \mathrm{CO} 2$ at $37^{\circ} \mathrm{C}$ for $15-30$ 
min in the dark. Then, the oocytes were washed twice with 1XPBS and imaged with LSCM using the fluorescein isothiocyanate (FITC, green) and rhodamine isothiocyanate (RITC, red) channels. The captured images (green and red) were acquired in the largest diameter plane of the oocytes using the confocal software. The ratio of RITC to FITC for each oocyte was calculated and used as the endpoint for the $\Delta \Psi \mathrm{m}$ [23].

\section{Next generation sequencing of arrested embryos}

Cleavage was assessed after $72 \mathrm{~h}$ post ICSI. Arrested cleaved embryos that were unable to develop to the blastocyst stage on day 5 , showed good quality and normal morphology with more than 4 cells in cleavage stage and obtained from zygotes with two pronuclei, were analyzed with NGS screening for whole chromosomes. Three groups of samples were analyzed: Group A, 12 arrested IVM embryos from 9 PCOS patients, Group B, 12 arrested IVM embryos from 10 non-PCOS patients, and Group C (control) 12 arrested IVF embryos randomly collected from 11 IVF patients less than 35 years old with the exclusion of ovarian insufficiency and male factor infertility that might be associated with paternal chromosomal abnormalities.

Briefly, the collected samples were washed in a PCR tube containing $2.5 \mu \mathrm{l}$ sterilized 1XPBS. Whole genomic amplification (WGA) of the samples was performed using the Sureplex DNA Amplification System (Blue Gnome, Cambridge, UK). WGA products were quantified using the Quan Tit dsDNA HS Assay Kit (Life Technologies Corporation, Grand Island, NY, USA), as reported previously $[24,25]$. Dual-indexed libraries were prepared using the Ion Xpress ${ }^{\text {mit }}$ Plus Fragment Library Kit (\# 4471269), the Ion Plus Fragment Library Kit (\# 4471252), and Ion Xpress $^{\text {Th }}$ Barcode Adapters 1-96 with the input sample DNA at $1 \mu \mathrm{g}$ gDNA (Illumina, San Diego, USA). Template-positive ISPs containing clonally amplified DNA were prepared by using the Ion PGM $^{\mathrm{m}}$ Template OneTouch $^{\text {Tx }} 2200$ Kit (\# 4480974), and sequencing with the Ion PGM ${ }^{\mathrm{sm}}$ Sequencing 200 Kit v2 (\# 4482006), Paired end, dual index $2 \times 36 \mathrm{bp}$ sequencing was performed using the Ion Torrent Proton (Life Technologies Corporation, Grand Island, USA).

\section{Statistical analysis}

Statistical analysis was performed using the SPSS version 21.0 software (SPSS, Chicago, IL). Differences between two groups were analyzed using unpaired $t$-tests. ANOVA was used for the analysis of multiple groups. Qualitative data were analyzed using the chi-square $\left(x^{2}\right)$ test. Data were represented as the mean $\pm \mathrm{SD}$, and a $P$ value $<0.05$ was considered statistically significant.

\section{Results}

Aneuploidy or chromosome abnormalities are the leading causes for embryo arrest, recurrent implantation failure, recurrent miscarriage, and birth defects that appear to be age-associated, and about $30-60 \%$ of embryos are reported to be aneuploid in patients older than 35 years of age [26-29]. To exclude age-associated embryo arrest, we selected patients who were less than 35 years old.

According to the Rotterdam criteria for phenotypes of PCOS patients [22], all PCOS patients recruited in our study were categorized as the non-hyperandrogenic PCOS phenotype (olig-anovulation and polycystic ovaries morphology) with normal endocrine test results and metabolic profiles. Patients with male factor infertility exhibited mild to moderate oligospermia (count $>5 \mathrm{M}$ / $\mathrm{ml}$ ), asthenospermia (progressive motility $<32 \%$ ), and abnormal morphology $<96 \%$. All patients with male infertility underwent screened chromosome analysis to exclude genetic abnormalities.

We did not observe any significant differences related to age, BMI, FSH levels on day 3 of the cycle, and duration of gonadotropin administrations between PCOS and non-PCOS patients as shown in Table 1.

Data measured by (mean $\pm \mathrm{SD})$, NS indicates a $P$-value $>$ 0.05 .

A total of 1375 oocytes were collected from all cycles from Group A (PCOS patients) and Group B (nonPCOS patients). Of these, at the time of retrieval, 168 MII (22.8\%), 473 GV (64.6\%), and 93 MI (12.6\%) oocytes were obtained from PCOS patients, and165 MII (25.7\%), 396 GV (61.7\%), and 80 MI (12.4\%) oocytes were obtained from non-PCOS patients. At $24 \mathrm{~h}$ of culture, the results showed the maturation rate in vitro was $63.6 \%$ vs. $54.6 \%$, with a total maturation rate of $68.9 \%$ vs. $58.8 \%$ in PCOS and non-PCOS groups, respectively.

There was no significant difference in the total rates of fertilization, cleavage, and blastocyst formation between the groups. When the efficiency of the IVM procedure was compared between the POCS and non-PCOS groups, the fertilization and blastocyst formation rates did not show significant differences between the two groups. However, the rate of oocyte in vitro maturation in Group A showed a small but significant difference $(P=0.04)$ compared to Group B (Table 2).

Mildly stimulated IVF/M cycles provided the patients with the chance for embryo transfer obtained from IVM oocytes and others obtained from in vivo MII oocytes. When a mean of 2 or 3 embryos was transferred, it resulted in cumulative clinical pregnancy rates of $40.2 \%$ vs. $41.9 \%$ and implantation rates of $28.7 \%$ vs. $36 \%$ in PCOS and non-PCOS groups, respectively. A fresh transfer of two cleavage embryos with IVM was performed in 44 IVM cycles. In addition, the transfer of frozen and thawed IVM blastocyst embryos (ET) was performed in 
Table 1 Characteristics of patients enrolled in our study

\begin{tabular}{|c|c|c|c|}
\hline Groups & $\mathrm{A}(\mathrm{PCOS})$ & B (non-PCOS) & $P$ value \\
\hline No of patients & 58 & 56 & \\
\hline Age & $28.0 \pm 3.26$ & $28.0 \pm 3.64$ & NS \\
\hline Body mass index (BMI) kg/m2 & $23.0 \pm 2.4$ & $22.0 \pm 1.7$ & NS \\
\hline FSH level & $5.0 \pm 1.2$ & $6.0 \pm 1.5$ & NS \\
\hline Days of gonadotropin stimulation & $4.0 \pm 1.5$ & $4.0 \pm 1.9$ & NS \\
\hline Tubal factor infertility & 35 & 37 & - \\
\hline Male factor infertility & 8 & 6 & - \\
\hline Tubal and male factor infertility & 5 & 5 & - \\
\hline Unexplained infertility & 10 & 8 & - \\
\hline
\end{tabular}

Data measured by (mean \pm SD), NS represent $(P$-value $>0.05)$

69 IVM cycles. Further, frozen and thawed ET of blastocysts obtained from in-vivo MII oocytes were transferred in 45 cycles.

For IVM/ET cycles, however, there were no significant differences between PCOS and non-PCOS patients with respect to fresh and frozen ET cycles. Thus, frozen and thawed ET cycles had a better outcome with respect to the clinical pregnancy rate and implantation rate in the two groups when compared with fresh ET cycles.

Frozen and thawed ET cycles for blastocysts obtained from in vivo MII oocytes yielded clinical pregnancy rates of $52.3 \%$ vs. $50 \%$ and implantation rates of $44 \%$ vs. $48 \%$ in PCOS and non-PCOS groups, respectively. No significant differences were observed between the two groups for biochemical pregnancy rate, miscarriage rate, and live birth rate. Seventeen patients delivered 14 healthy boys, and 5 girls, and 43 pregnancies were still ongoing at the time of manuscript preparation (Table 3).

MII oocytes were stained with the mitochondria-specific probe, JC-1, and visualized with LSCM, a red fluorescent stained, which located around the periphery of the oocyte indicates higher mitochondrial membrane potential. While, green fluorescence is dispersed within the oocyte cytoplasm indicates lower mitochondrial membrane potential. We found that oocytes matured in vitro presented reduced red fluorescence in periphery of the cell compared to oocytes that were matured in vivo; the green fluorescence had no observable change (Fig. 1a). We investigated the high polarization of mitochondria in MII oocytes by examining the relative ratio of emitted red to green fluorescence. The ratio of red to green $\mathrm{JC}-1$ fluorescence in MII oocytes from IVM patients sharply decreased, compared with the control group C (MII oocytes from IVF patients, $P<0.001$ ), which indicated that the $\Delta \Psi \mathrm{m}$ was decreased without differences between the IVM groups (PCOS and non-PCOS) $(P=0.7)$ as shown in Fig. 1b.

Next generation sequencing (NGS) is a recent technology used for preimplantation genetic diagnosis [30], and it was employed to analyze 36 arrested cleaved embryos obtained from our cycles (Fig. 2). Our findings reveled that the aneuploidy rates in arrested embryos of IVMPCOS, IVM-non PCOS, control IVF were (75, 75, and $66.6 \%)$, respectively, with no significant difference among the three groups as shown in Table 4. Furthermore, the different types of chromosomal abnormalities such as monosomy, dual, trisomy, complex, and mosaicism were observed in the three groups and suggested that aneuploidy is not the possible cause for embryo arrest.

\section{Discussion}

Standard IVF is superior to IVM in terms of the clinical pregnancy rate, implantation rate, and number and quality of embryos. However, the IVM procedure has physical, physiological, and financial advantages. Although IVM is indicated to avoid ovarian hyper stimulation syndrome (OHSS) in PCOS patients and recently used for fertility preservation in cancer patients, it is still in its an underutilized procedure due to its low efficiency. It is still not known whether the low efficiency is due to the PCOS condition or the IVM itself.

To investigate the IVM efficiency, we administered FSH combined with hCG priming to improve the nuclear maturation rate and increase the number of retrieved mature oocytes compared to non-hCG primed cycles, while the FSH priming improved the competence of oocytes for proper development [31]. Our experience has shown that it is easier to retrieve more oocytes from follicles around $10 \mathrm{~mm}$ in diameter compared to smaller follicles.

In line with Lim et al. [3], mildly stimulated IVM cycles with FSH combined hCG priming resulted in 22.8 and $25.7 \%$ mature oocytes from small follicles less than $12 \mathrm{~mm}$ in diameter in PCOS and nonPCOS groups, respectively. This might confirm the presence of LH or hCG receptors in the cumulus and granulosa cells from the small follicles [32]. Even 
Table 2 Lab outcomes of mildly stimulated IVF/M cycle in Group A (PCOS) and Group B (non-PCOS) patients

\begin{tabular}{|c|c|c|c|}
\hline Groups & A (PCOS) $(n=58)$ & B (non-PCOS) $(n=56)$ & $P$ value \\
\hline No. of mature oocytes retrieved (mean \pm SD) & $168(2.9 \pm 5)$ & $165(2.8 \pm 4.1)$ & NS \\
\hline No. of in vivo mature oocytes fertilized (mean \pm SD) & $126(2.1 \pm 3.6)$ & $128(2.2 \pm 3.6)$ & NS \\
\hline No. of in vivo mature oocytes cleaved (mean \pm SD) & $107(1.8 \pm 3.2)$ & $111(1.9 \pm 3.1)$ & NS \\
\hline No. of blastocyst from in vivo mature oocytes (mean \pm SD) (\%) & $72(1.2 \pm 2.1)(57.1 \%)$ & $80(1.3 \pm 2.2)(62.5 \%)$ & NS \\
\hline No. of immature oocytes retrieved (mean \pm SD) & $566(9 \pm 5.7)$ & $476(8 \pm 5.3)$ & NS \\
\hline No. of oocytes matured in vitro (\%) & $390(68.9 \%)$ & $280(58.8 \%)$ & 0.04 \\
\hline No. of in vitro mature oocytes fertilized (mean \pm SD) (\%) & $285(4.6 \pm 3.1)(73 \%)$ & $205(3.5 \pm 2.9)(73.2 \%)$ & NS \\
\hline No. of in vitro mature oocytes cleaved (mean \pm SD) & $226(3.6 \pm 2.7)$ & $152(2.5 \pm 2.2)$ & NS \\
\hline No. of blastocyst from in vitro mature oocytes (mean \pm SD) (\%) & $80(1.3 \pm 1.2)(28 \%)$ & $58(0.9 \pm 1.1)(28.2 \%)$ & NS \\
\hline Total no. of oocytes matured (mean \pm SD) & $558(9 \pm 5.7)$ & $445(7 \pm 4.7)$ & NS \\
\hline Total no. of oocytes fertilized (\%) & $411(73.6 \%)$ & $333(74.8 \%)$ & NS \\
\hline Total no. of zygote cleaved (\%) & $333(81 \%)$ & $263(78.9 \%)$ & NS \\
\hline Total no. of blastocyst (\%) & $152(36.9 \%)$ & $138(41.4 \%)$ & NS \\
\hline
\end{tabular}

Data measured by (mean \pm SD) and percentage $(\%)$, NS represent $(P$-value $>0.05)$

so, it is unclear how the small follicles react to the LH surge.

The non-hCG primed IVM cycles yielded a fewer number of retrieved mature oocytes compared to the hCG primed cycles. Researchers thought that these mature oocytes had completed the nuclear maturation while the cytoplasmic maturation was not completed. This lack of synchronization resulted in lower fertilization and blastocyst formation rates compared to standard IVF $[14,33]$. However, in this study, the mature oocytes with FSH and hCG priming were capable of being fertilized $(75,77.5 \%)$ and developed to blastocyst embryos $(57.1,62.5 \%)$ in PCOS and non-PCOS groups, respectively, as shown in Table 2, and even the size of these follicles was less than the size in standard IVF protocols.

In line with Chian et al., the developmental competence of immature oocytes from small follicles was not impaired by the presence of mature follicles [2]. In addition, it was reported that the clinical pregnancy rate was higher in the IVF/M cycle with in vivo matured oocytes $(40.0 \%$ vs. 23.3\%) compared to the IVM cycles with immature oocytes only [3,34]. These observations support our findings that mildly stimulated IVF/M cycles could achieve a higher cumulative clinical pregnancy rate and implantation rate $(40.2,28.7 \%$ in the PCOS group vs. $41.9,36 \%$ in the non-PCOS group), respectively.

Most researchers believed that PCOS patients are the ideal group to undergo the IVM procedure due to a large number of antral follicles, oocyte retrieved, and blastomeres. It also is the case that PCOS patients showed better clinical and laboratory IVM outcomes compared to the non-PCOS group [35, 36]. Our findings showed no significant differences in the number of oocytes retrieved, rates of in vitro maturation, fertilization, cleavage, and blastocyst formation in PCOS and nonPCOS patients.

For our clinical outcome in IVM/ET cycles, however, the difference between the two groups was nonsignificant, the non-PCOS group showed better outcomes in the cumulative clinical pregnancy rate $(38.5 \%$ vs. $35.7 \%)$, implantation rate $(32.0 \%$ vs. $24.1 \%)$, biochemical pregnancy ( $43.2 \%$ vs. $42.8 \%)$ and miscarriage rate $(5.8 \%$ vs. 9\%) compared to the PCOS group, respectively. The latter group showed a comparable cumulative clinical pregnancy of $35.7 \%$ with the outcome of $35.0 \%$ for ICSI and IVM cycles in PCOS reported by Walls et al. [15].

Our findings showed a lower outcome efficiency compared with the non-hCG primed IVM cycles reported in the Walls et al. study, which compared the outcome of 98 IVF cycles with 80 cycles of non-hCG primed IVM treatment in PCOS patients. They found that the cumulative clinical pregnancy and implantation rates were nearly $51 \%$ and live birth rate was $41.3 \%$ [14], which was consistent with other studies that used similar approaches $[9,37]$. These high rates of cumulative clinical pregnancy and implantation may be due to the transfer of blastocyst embryos in fresh and frozen ET cycles. In our study, frozen ET cycles of IVM blastocysts yielded nearly the same clinical pregnancy (37.1-47.1\%) and implantation rates $(28.2-45 \%)$ as the Walls report of frozen ET cycles (35.5, 44\%) [14].

While our findings were comparable to Zheng's randomized controlled study that investigated the effect of hCG priming in IVM cycles in 82 PCOS patients who underwent Day3 ET cycles. They found that the rate of in vitro maturation was significantly higher in the hCGprimed group ( $55.4 \%$ vs. $42.2 \% ; p<0.05$ ) than the nonhCG primed group with comparable fertilization rates $(63.4 \%$ vs. $65.4 \%)$ between the groups. There were no 
Table 3 Clinical outcome of mildly stimulated IVF/M cycle in Group A (PCOS) and Group B (non-PCOS) patients

\begin{tabular}{|c|c|c|c|}
\hline Groups & $\begin{array}{l}\text { A (PCOS) } \\
(n=58)\end{array}$ & $\begin{array}{l}\text { B (non-PCOS) } \\
(n=56)\end{array}$ & $P$ value \\
\hline No. of IVM/ET cycles & 56 & 57 & NS \\
\hline \multicolumn{4}{|l|}{ Clinical pregnancy rate } \\
\hline Cumulative rate & (20/56) $35.7 \%$ & $(22 / 57) 38.5 \%$ & NS \\
\hline Fresh ET rate & $(7 / 21) 33.3 \%$ & (6/23) $26.1 \%$ & NS \\
\hline Frozen ET rate & (13/35) $37.1 \%$ & $(16 / 34) 47.1 \%$ & NS \\
\hline No. of embryo transferred & 83 & 75 & NS \\
\hline No. of embryo implanted & 20 & 24 & NS \\
\hline \multicolumn{4}{|l|}{ Implantation rate } \\
\hline Cumulative rate & (20/83) $24.1 \%$ & $(24 / 75) 32 \%$ & NS \\
\hline Fresh ET rate & $(7 / 37) 18.9 \%$ & $(6 / 35) 17.1 \% *$ & NS \\
\hline Frozen ET rate & $(13 / 46) 28.2 \%$ & $(18 / 40) 45 \% *$ & NS \\
\hline No. of IVF/ET cycles & 21 & 24 & \\
\hline Clinical pregnancy rate & $(11 / 21) 52.3 \%$ & (12/24) 50\% & NS \\
\hline Implantation rate & $(11 / 25) 44 \%$ & $(12 / 25) 48 \%$ & NS \\
\hline Total no. of ET cycles & 77 & 81 & \\
\hline Cumulative pregnancy rate & (31/77) $40.2 \%$ & (34/81) 41.9\% & NS \\
\hline Cumulative implantation rate & $(31 / 108) 28.7 \%$ & $(36 / 100) 36 \%$ & NS \\
\hline No. of biochemical pregnancy & $(33 / 77)(42.8 \%)$ & $(35 / 81)(43.2 \%)$ & NS \\
\hline No. of miscarriage & $(3 / 33)(9 \%)$ & )2/35) (5.8\%) & NS \\
\hline No. of livebirth & 9 & 10 & NS \\
\hline No. of ongoing pregnancy & 20 & 23 & \\
\hline
\end{tabular}

Data measured by percentage $(\%)$; NS represent $(P$-value $>0.05)$

N.B: * represent significant difference between implantation rate of fresh and frozen ET in group B $(P<0.05)$

significant differences in the clinical pregnancy rate $(37.5 \%$ vs. $50 \%)$, live birth rate $(22.5 \%$ vs. $30 \%)$, and implantation rate $(32.8 \%$ vs. $32.5 \%)$ between the two groups [38].

Our findings showed better clinical pregnancy and implantation rates in frozen ET cycles compared to fresh ET cycles in PCOS and non-PCOS groups. This might be related to the stage of embryo that was transferred, since blastocyst embryos were transferred in frozen ET cycle while cleavage embryos were transferred in fresh ET cycles. According to natural selection, only good quality cleavage embryos have the ability to develop to the blastocyst stage. Thus, this might be related to asynchrony between the state of the endometrium and the IVM embryos.

Additionally, the blastocyst formation rates from IVM oocytes, an indirect measure for early embryo arrest, were comparable in PCOS and non-PCOS patients (28\% vs $28.2 \%$, respectively) while the blastocyst formation rate from in vivo MII oocytes were $57.1 \%$ vs 62.5 , respectively, as shown in Table 2 . However, the size of in vivo MII oocytes that were retrieved was less than 12 $\mathrm{mm}$ in diameter, but the blastocyst formation rate was still higher than that of IVM oocytes. However, Walls reported that the blastocyst formation rate in PCOSIVM embryos was comparatively high (48.2\%) [15]. Unfortunately, it was associated with an increased rate of early embryo arrest during the third cytokinesis (Day 34 stage) compared to the ICSI group [15]. Altogether these findings suggested that the IVM itself is the possible cause of early embryo arrest rather than the PCOS condition. In conclusion, a better understanding and improvement of IVM procedure is still needed.

The variety of factors that increased the possibility of early embryo arrest include genetic causes, oxidative stress, aneuploidy, energy stores defects [19, 39, 40], and mitochondrial dysfunction through $\Delta \Psi \mathrm{m}$ that drives the conversion of ADP to ATP by respiratory chain enzymes [41]. Recently, Lei et al. found that cryopreservation significantly decreased the inner mitochondrial membrane potential of vitrified IVM oocytes compared to fresh IVM oocytes $(P<0.05)[42]$. For the first time, we compared the mitochondrial membrane potential of IVM and IVF oocytes. Using LSCM, we found a significant decrease in the $\Delta \Psi \mathrm{m}$ of IVM oocytes compared to control-IVF group $(P<0.001)$ with no difference between PCOS and non-PCOS groups $(P=0.7)$. 


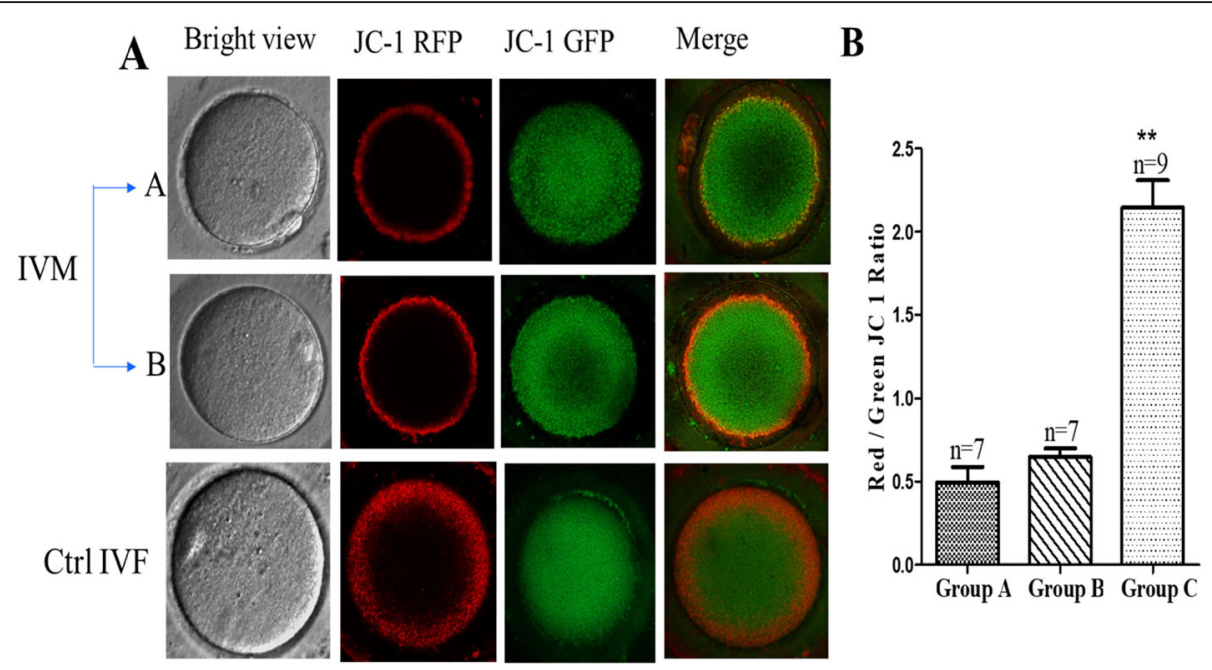

Fig. 1 Effect of IVM on the inner mitochondrial membrane potential $(\Delta \psi \mathrm{m})$ measured by JC-1 fluorescence, a: Relative inner membrane potential in MII oocytes from A: IVM-PCOS, B: IVM-non PCOS and C: control IVF groups. b: Ratio of red to green JC-1 fluorescence in the three groups. Data measured by mean $\pm \mathrm{SD}$, ** represent $P<0.05$

Unlike in vivo maturation, which was accompanied by increased cAMP levels, which in turn, induced epidermal growth factor (EGF)-like peptide expression in cumulus cells leading to cumulus expansion and oocyte maturation, in vitro maturation of oocytes is a spontaneous process due to decreased EGF-like peptides that result from a sudden drop in immature COC cAMP [43-46], which might account for the lower mitochondrial function.
However, FSH stimulates and maintains EGF-like peptide expression in vivo, which was unable to do so when added to the IVM media. On the other hand, in animal studies, supplementation with EGF-like peptides significantly increased mitochondrial activity in oocytes matured in vitro through enhanced glucose metabolism and protein glycosylation, which in turn, increased the developmental competence of oocytes and blastocyst formation rate $[47,48]$. Also, the pre-maturation stage with

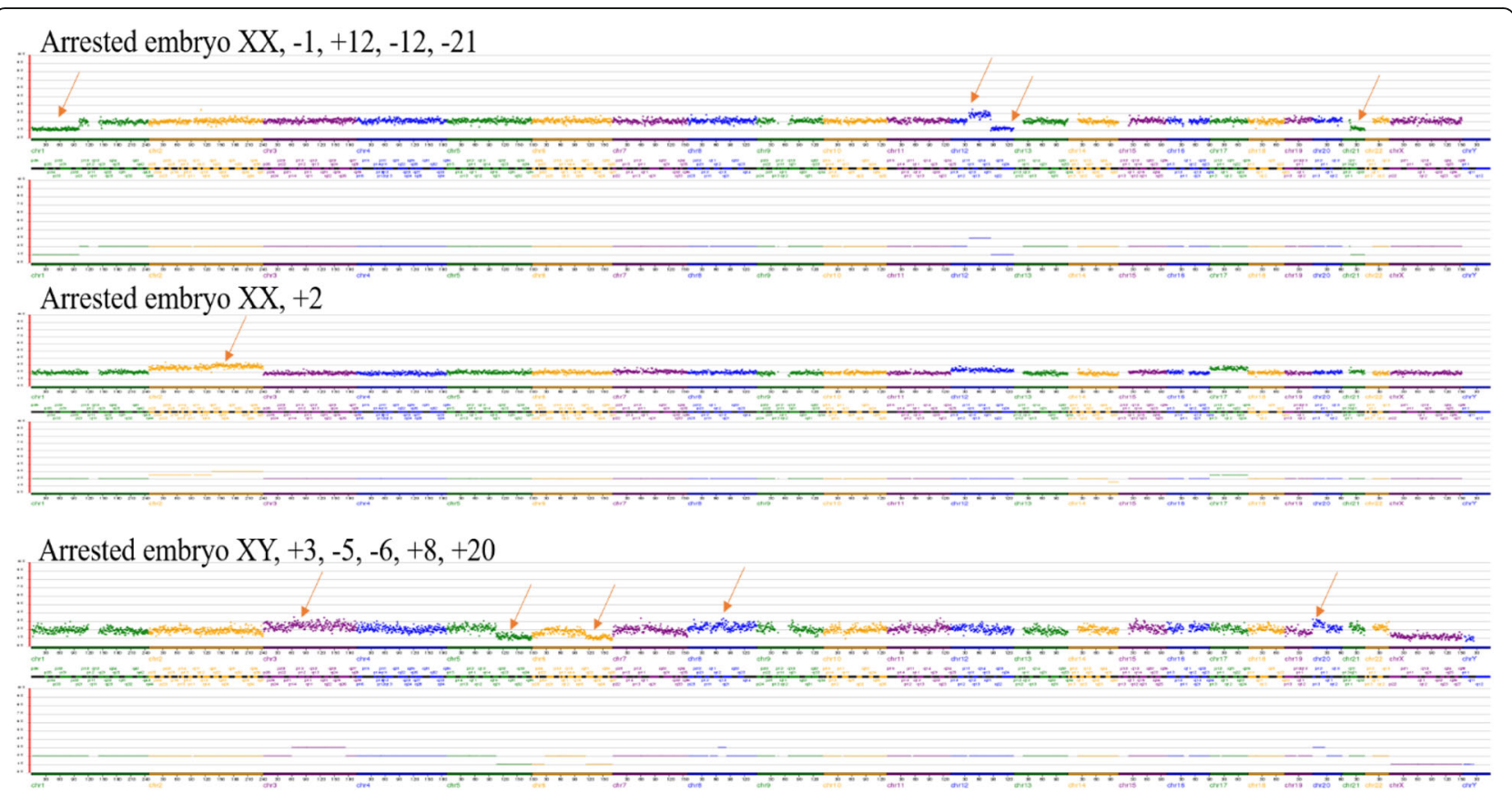

Fig. 2 NGS charts of arrested embryos with different types of chromosomal abnormalities, Notes: Arrows indicate chromosome errors. 
Table 4 Next generation sequencing determinations for euploidy and aneuploidy in arrested embryos from IVM-PCOS, IVM-nonPCOS, and control IVF groups

\begin{tabular}{lcccc}
\hline Groups & $\begin{array}{c}\text { A (IVM-PCOS) } \\
\mathbf{n}=\mathbf{1 2}\end{array}$ & $\begin{array}{c}\text { B (IVM-non } \\
\text { PCOS) } \mathbf{n}=\mathbf{1 2}\end{array}$ & $\begin{array}{c}\text { C (IVF) } \\
\mathbf{n = 1 2}\end{array}$ & P value \\
\hline Euploid rate & $3 / 12(25 \%)$ & $3 / 12(25 \%)$ & $4 / 12(33.3 \%)$ & NS \\
\hline Aneuploid rate & $9 / 12(75 \%)$ & $9 / 12(75 \%)$ & $8 / 12(66.6 \%)$ & NS \\
\hline Monosomy & $2 / 9(22.2 \%)$ & $2 / 9(22.2 \%)$ & $0 / 8(0 \%)$ & \\
\hline Dual & $3 / 9(33.3 \%)$ & $2 / 9(22.2 \%)$ & $1 / 8(12.5 \%)$ & \\
\hline Trisomy & $1 / 9(11.1 \%)$ & $2 / 9(22.2 \%)$ & $4 / 8(50 \%)$ & \\
\hline Complex & $1 / 9(11.1 \%)$ & $2 / 9(22.2 \%)$ & $2 / 8(25 \%)$ & \\
\hline Mosaicism & $2 / 9(22.2 \%)$ & $1 / 9(11.1 \%)$ & $1 / 8(12.5 \%)$ \\
\hline
\end{tabular}

Data measured by percentage (\%); NS indicates $P$ value $>0.05$

cAMP modulators and EGF-like peptides in the IVM technique could attenuate spontaneous oocyte maturation and extend cumulus oocyte gap-junction, resulting in a significant increase in oocyte developmental competence $[46,49,50]$.

Based on the above evidence, the IVM-associated high rate of early embryonic arrest might be attributed to mitochondrial dysfunction. Thus, further laboratory investigations with large samples in human are still needed to improve the clinical efficiency of mitochondrial nutrients that can be added to IVM culture medium.

Regarding the consequence of the higher aneuploidy rate in arrested embryos, Previous studies reported that nearly $70 \%$ of arrested and slowly cleaving embryos had chromosomal abnormalities, which suggested aneuploidy was a major cause of embryonic arrest [21, 51, 52]. To exclude the possible effect of ovarian hyperstimulation on the aneuploidy rate, Labarta et al. found that moderate ovarian stimulation did not significantly increase the aneuploidy rate [53].

Using FISH, Requena et al. revealed a $60 \%$ aneuploidy rate in IVM embryos compared to $33 \%$ in control groups [54]. While, Benkhalifa et al. found a 32.6-49.2\% aneuploidy rate in IVM arrested embryos from PCOS patients [55]. Although the incidence of aneuploidy greatly varies in the two studies, the smaller number of chromosomes screened, a main limitation of FISH, different IVM protocols that were applied, and variations between participants (normal oocyte donors and PCOS women) may account for the observed differences. Recently, using LSCM, several studies reported that in vitro culture had no impact on the IVM efficiency or the frequency of meiotic anomalies in POCS patients compared to controls, but they reported an increase in the percent of meiotic anomalies, which suggested that IVM itself might cause an increase in the incidence of meiotic abnormalities that then resulted in chromosomal disorders [56, 57].

We investigated the aneuploidy rates in arrested (IVM and IVF) embryos using NGS on small DNA fragments that covers the whole chromosomes [30]. The sequencing results showed that the aneuploidy rates were comparable $(75,75$, and $66.6 \%)$ in IVM-PCOS, IVM-non PCOS, and control IVF arrested embryos, respectively. Our results were comparable to Zhang's retrospective study on chromosomal abnormalities in IVM and IVF embryos (58.7\% vs. $57.4 \%$, respectively) [58]. Therefore, aneuploidy does not appear to be the predisposing reason for the high incidence of early embryo arrest in IVM cycles.

\section{Conclusions}

Mild stimulated IVF/M cycles can be an attractive choice for normal ovulatory and anovulatory PCOS patients not only to avoid OHSS but also due to its simplified patient-friendly ART procedure that has lower cost and acceptable clinical pregnancy rates. The current study reported no significant differences in the laboratory and clinical outcomes of the IVM procedure in PCOS and non-PCOS patients, which encourage clinicians to apply such technique for non-PCOS as well PCOS patients. In addition, frozen and thawed ET cycles had a higher clinical pregnancy and implantation rate compared to that of fresh ET cycles. Thus, it is necessary to pay more attention to the possibility of asynchrony between the endometrium and the embryos. 
Additionally, we found that embryo arrest is IVM procedure-related rather than due to the PCOS condition itself. As seen with using LSCM, MII oocytes after IVM culture showed decreased mitochondrial membrane potentials compared to in-vivo MII oocytes, but with no difference between PCOS and non-PCOS patients. NGS showed comparable aneuploidy rates of arrested IVM/IVF embryos. Therefore, we concluded that IVM itself adversely affected oocyte and embryo development by affecting mitochondrial function that seemed to be the possible cause for lower blastocyst formation rates in IVM cycles. Further laboratory investigations with larger sample sizes are still needed to improve IVM oocyte and embryo competence in vitro culturing.

\section{Acknowledgements}

We would like to thank all the staff members of the reproductive medical center of the first affiliated Hospital of Anhui medical university, Hefei, China. We are grateful for the assistsance of Dr. Louise Abbott (Professor At Texas A\&M (TAMU) in editting of this manuscript.

\section{Authors' contributions}

All authors read and approved the final manuscript.

\section{Funding}

This study was supported by the National Key R\&D Program of China (2017YFC1001300) and the National Natural Science Foundation of China $(81601345,81871216)$

\section{Availability of data and materials}

Not applicable.

\section{Ethics approval and consent to participate}

This study was approved by the committee on biological ethics of Anhui Medical University, Hefei, China (No. 20160097). All patients received a full explanation of the mildly stimulated IVF/M protocol and provided written signed informed consent. Also, all donor patients of MII oocytes (after IVM or IVF) signed the written informed consent.

\section{Consent for publication}

Not applicable.

\section{Competing interests}

The authors declare that they have no competing interests.

\begin{abstract}
Author details
${ }^{1}$ Reproductive Medicine Center, Department of Obstetrics and Gynecology, the First Affiliated Hospital of Anhui Medical University, Hefei, China. ${ }^{2}$ Key Laboratory of Population Health Across Life Cycle (Anhui Medical University), Ministry of Education of the People's Republic of China, Hefei, China. ${ }^{3}$ Anhui Province Key Laboratory of Reproductive Health and Genetics, Hefei, China. ${ }^{4} \mathrm{NHC}$ Key Laboratory of Study on Abnormal Gametes and Reproductive Tract, Anhui Medical University, Hefei, China. ${ }^{5}$ Anhui Provincial Engineering Technology Research center for Bio preservation and Artificial Organs, Hefei, China.
\end{abstract}

Received: 17 February 2020 Accepted: 18 June 2020 Published online: 15 July 2020

\section{References}

1. Dahan MH, Tan SL, Chung J, Son WY. Clinical definition paper on in vitro maturation of human oocytes. Hum Reprod. 2016;31:1383-6.

2. Chian RC, Buckett WM, Abdul Jalil AK, Son WY, Sylvestre C, Rao D, Tan SL. Natural-cycle in vitro fertilization combined with in vitro maturation of immature oocytes is a potential approach in infertility treatment. Fertil Steril. 2004;82:1675-8.
3. Lim JH, Yang SH, Chian RC. New alternative to infertility treatment for women without ovarian stimulation. Reprod BioMed Online. 2007;14:547-9.

4. Son WY, Tan SL. Laboratory and embryological aspects of hCG-primed in vitro maturation cycles for patients with polycystic ovaries. Hum Reprod Update. 2010;16:675-89.

5. Chian RC, Lim JH, Tan SL. State of the art in in-vitro oocyte maturation. Curr Opin Obstet Gynecol. 2004;16:211-9.

6. Siristatidis CS, Vrachnis N, Creatsa M, Maheshwari A, Bhattacharya S. In vitro maturation in subfertile women with polycystic ovarian syndrome undergoing assisted reproduction. Cochrane Database Syst Rev. 2013: CD006606.

7. Farsi MM, Kamali N, Pourghasem M. Embryological aspects of oocyte in vitro maturation. Int J Mol Cell Med. 2013;2:99-109.

8. Buckett WM, Chian RC, Tan SL. Human chorionic gonadotropin for in vitro oocyte maturation: does it improve the endometrium or implantation? J Reprod Med. 2004;49:93-8.

9. Junk SM, Yeap D. Improved implantation and ongoing pregnancy rates after single-embryo transfer with an optimized protocol for in vitro oocyte maturation in women with polycystic ovaries and polycystic ovary syndrome. Fertil Steril. 2012;98:888-92.

10. Vitek WS, Witmyer J, Carson SA, Robins JC. Estrogen-suppressed in vitro maturation: a novel approach to in vitro maturation. Fertil Steril. 2013;99: 1886-90.

11. Reavey J, Vincent K, Child T, Granne IE. Human chorionic gonadotrophin priming for fertility treatment with in vitro maturation. Cochrane Database Syst Rev. 2016;11:CD008720.

12. Shalom-Paz E, Holzer H, Son W, Levin I, Tan SL, Almog B. PCOS patients can benefit from in vitro maturation (IVM) of oocytes. Eur J Obstet Gynecol Reprod Biol. 2012;165:53-6.

13. Ellenbogen A, Shavit T, Shalom-Paz E. IVM results are comparable and may have advantages over standard IVF. Facts Views Vis Obgyn. 2014;6:77-80.

14. Walls ML, Hunter T, Ryan JP, Keelan JA, Nathan E, Hart RJ. In vitro maturation as an alternative to standard in vitro fertilization for patients diagnosed with polycystic ovaries: a comparative analysis of fresh, frozen and cumulative cycle outcomes. Hum Reprod. 2015;30:88-96.

15. Walls ML, Ryan JP, Keelan JA, Hart R. In vitro maturation is associated with increased early embryo arrest without impairing morphokinetic development of useable embryos progressing to blastocysts. Hum Reprod. 2015;30:1842-9.

16. Wissing ML, Bjerge MR, Olesen Al, Hoest $\mathrm{T}$, Mikkelsen AL. Impact of PCOS on early embryo cleavage kinetics. Reprod BioMed Online. 2014; 28:508-14.

17. Rubio I, Galan A, Larreategui Z, Ayerdi F, Bellver J, Herrero J, Meseguer M. Clinical validation of embryo culture and selection by morphokinetic analysis: a randomized, controlled trial of the EmbryoScope. Fertil Steril. 2014;102:1287-94 e1285.

18. Schoolcraft WB, Katz-Jaffe MG, Stevens J, Rawlins M, Munne S. Preimplantation aneuploidy testing for infertile patients of advanced maternal age: a randomized prospective trial. Fertil Steril. 2009;92:157-62.

19. Agarwal A, Aponte-Mellado A, Premkumar BJ, Shaman A, Gupta S. The effects of oxidative stress on female reproduction: a review. Reprod Biol Endocrinol. 2012;10:49.

20. Van Blerkom J. Mitochondrial function in the human oocyte and embryo and their role in developmental competence. Mitochondrion. 2011;11:797-813.

21. Maurer M, Ebner T, Puchner M, Mayer RB, Shebl O, Oppelt P, Duba HC. Chromosomal aneuploidies and early embryonic developmental arrest. Int J Fertil Steril. 2015:9:346-53.

22. Rotterdam EA-SPcwg. Revised 2003 Consensus on diagnostic criteria and long-term health risks related to polycystic ovary syndrome (PCOS). Hum Reprod. 2004;19:41-7.

23. Wilding $M$, Dale $B$, Marino $M$, di Matteo L, Alviggi C, Pisaturo ML, Lombardi L, De Placido G. Mitochondrial aggregation patterns and activity in human oocytes and preimplantation embryos. Hum Reprod. 2001;16:909-17.

24. Gutierrez-Mateo C, Colls P, Sanchez-Garcia J, Escudero T, Prates R, Ketterson K, Wells D, Munne S. Validation of microarray comparative genomic hybridization for comprehensive chromosome analysis of embryos. Fertil Steril. 2011;95:953-8.

25. Yang Z, Liu J, Collins GS, Salem SA, Liu X, Lyle SS, Peck AC, Sills ES, Salem $\mathrm{RD}$. Selection of single blastocysts for fresh transfer via standard morphology assessment alone and with array CGH for good prognosis IVF patients: results from a randomized pilot study. Mol Cytogenet. 2012;5:24. 
26. Hodes-Wertz B, Grifo J, Ghadir S, Kaplan B, Laskin CA, Glassner M, Munne S Idiopathic recurrent miscarriage is caused mostly by aneuploid embryos. Fertil Steril. 2012;98:675-80.

27. Harton GL, Munne S, Surrey M, Grifo J, Kaplan B, McCulloh DH, Griffin DK, Wells D, Group PGDP. Diminished effect of maternal age on implantation after preimplantation genetic diagnosis with array comparative genomic hybridization. Fertil Steril. 2013;100:1695-703.

28. Franasiak JM, Forman EJ, Hong KH, Werner MD, Upham KM, Treff NR, Scott RT Jr. The nature of aneuploidy with increasing age of the female partner: a review of 15,169 consecutive trophectoderm biopsies evaluated with comprehensive chromosomal screening. Fertil Steril. 2014;101:656-63 e651.

29. Sills ES, Li X, Frederick JL, Khoury CD, Potter DA. Determining parental origin of embryo aneuploidy: analysis of genetic error observed in 305 embryos derived from anonymous donor oocyte IVF cycles. Mol Cytogenet. 2014;7:68.

30. Yin X, Tan K, Vajta G, Jiang H, Tan Y, Zhang C, Chen F, Chen S, Zhang C, Pan $X$, et al. Massively parallel sequencing for chromosomal abnormality testing in trophectoderm cells of human blastocysts. Biol Reprod. 2013;88:69.

31. Wang P, Zhao J, Jin C, Yu R, Lin J, Zhu R. Wu Y: [Comparision of in vitro maturation applied in PCOS and non-PCOS patients undergo stimulated and unstimulated protocols]. Zhonghua Fu Chan Ke Za Zhi. 2014:49:903-8.

32. Yang SH, Son WY, Yoon SH, Ko Y, Lim JH. Correlation between in vitro maturation and expression of $\mathrm{LH}$ receptor in cumulus cells of the oocytes collected from PCOS patients in HCG-primed IVM cycles. Hum Reprod. 2005; 20:2097-103.

33. Gremeau AS, Andreadis N, Fatum M, Craig J, Turner K, McVeigh E, Child T. In vitro maturation or in vitro fertilization for women with polycystic ovaries? A case-control study of 194 treatment cycles. Fertil Steril. 2012;98:355-60.

34. Son WY, Chung JT, Demirtas E, Holzer H, Sylvestre C, Buckett W, Chian RC, Tan SL. Comparison of in-vitro maturation cycles with and without in-vivo matured oocytes retrieved. Reprod BioMed Online. 2008;17:59-67.

35. Siristatidis C, Sergentanis TN, Vogiatzi P, Kanavidis P, Chrelias C, Papantoniou $\mathrm{N}$, Psaltopoulou T. In Vitro Maturation in Women with vs without Polycystic Ovarian Syndrome: A Systematic Review and Meta-Analysis. PLoS One. 2015; 10:e0134696.

36. Tannus S, Hatirnaz S, Tan J, Ata B, Tan SL, Hatirnaz E, Kenat-Pektas M, Dahan $\mathrm{MH}$. Predictive factors for live birth after in vitro maturation of oocytes in women with polycystic ovary syndrome. Arch Gynecol Obstet. 2018;297: 199-204.

37. Romero S, Pella R, Escudero F, Perez Y, Garcia M, Orihuela P. Occurrence of ovarian follicular dominance during stimulation for IVM impacts usable blastocyst yield. JBRA Assist Reprod. 2018;22:56-60.

38. Zheng X, Wang L, Zhen X, Lian Y, Liu P, Qiao J. Effect of hCG priming on embryonic development of immature oocytes collected from unstimulated women with polycystic ovarian syndrome. Reprod Biol Endocrinol. 2012;10:40.

39. Favetta LA, St John EJ, King WA, Betts DH. High levels of p66shc and intracellular ROS in permanently arrested early embryos. Free Radic Biol Med. 2007:42:1201-10.

40. Passos JF, Saretzki G, Ahmed S, Nelson G, Richter T, Peters H, Wappler I, Birket MJ, Harold G, Schaeuble K, et al. Mitochondrial dysfunction accounts for the stochastic heterogeneity in telomere-dependent senescence. PLoS Biol. 2007:5:e110.

41. Van Blerkom J, Davis P, Mathwig V, Alexander S. Domains of high-polarized and low-polarized mitochondria may occur in mouse and human oocytes and early embryos. Hum Reprod. 2002;17:393-406.

42. Lei T, Guo N, Liu JQ, Tan MH, Li YF. Vitrification of in vitro matured oocytes: effects on meiotic spindle configuration and mitochondrial function. Int J Clin Exp Pathol. 2014;7:1159-65.

43. Guzman L, Adriaenssens T, Ortega-Hrepich C, Albuz FK, Mateizel I, Devroey P, De Vos M, Smitz J. Human antral follicles $<6 \mathrm{~mm}$ : a comparison between in vivo maturation and in vitro maturation in non-hCG primed cycles using cumulus cell gene expression. Mol Hum Reprod. 2013;19:7-16.

44. Kind KL, Banwell KM, Gebhardt KM, Macpherson A, Gauld A, Russell DL, Thompson JG. Microarray analysis of mRNA from cumulus cells following in vivo or in vitro maturation of mouse cumulus-oocyte complexes. Reprod Fertil Dev. 2013;25:426-38.

45. Vaccari S, Weeks JL 2nd, Hsieh M, Menniti FS, Conti M. Cyclic GMP signaling is involved in the luteinizing hormone-dependent meiotic maturation of mouse oocytes. Biol Reprod. 2009;81:595-604.

46. Albuz FK, Sasseville M, Lane M, Armstrong DT, Thompson JG, Gilchrist RB. Simulated physiological oocyte maturation (SPOM): a novel in vitro maturation system that substantially improves embryo yield and pregnancy outcomes. Hum Reprod. 2010;25:2999-3011.

47. Richani D, Sutton-McDowall ML, Frank LA, Gilchrist RB, Thompson JG. Effect of epidermal growth factor-like peptides on the metabolism of in vitromatured mouse oocytes and cumulus cells. Biol Reprod. 2014;90:49.

48. Richani D, Ritter LJ, Thompson JG, Gilchrist RB. Mode of oocyte maturation affects EGF-like peptide function and oocyte competence. Mol Hum Reprod. 2013;19:500-9.

49. Zeng HT, Ren Z, Guzman L, Wang X, Sutton-McDowall ML, Ritter L, De Vos M, Smitz J, Thompson JG, Gilchrist RB. Heparin and CAMP modulators interact during pre-in vitro maturation to affect mouse and human oocyte meiosis and developmental competence. Hum Reprod. 2013;28:1536-45.

50. Richani D, Wang X, Zeng HT, Smitz J, Thompson JG, Gilchrist RB. Prematuration with CAMP modulators in conjunction with EGF-like peptides during in vitro maturation enhances mouse oocyte developmental competence. Mol Reprod Dev. 2014;81:422-35.

51. Magli MC, Gianaroli L, Ferraretti AP, Lappi M, Ruberti A, Farfalli V. Embryo morphology and development are dependent on the chromosomal complement. Fertil Steril. 2007:87:534-41.

52. Demko ZP, Simon AL, McCoy RC, Petrov DA, Rabinowitz M. Effects of maternal age on euploidy rates in a large cohort of embryos analyzed with 24-chromosome single-nucleotide polymorphism-based preimplantation genetic screening. Fertil Steril. 2016;105:1307-13.

53. Labarta E, Bosch E, Alama P, Rubio C, Rodrigo L, Pellicer A. Moderate ovarian stimulation does not increase the incidence of human embryo chromosomal abnormalities in in vitro fertilization cycles. J Clin Endocrinol Metab. 2012;97:E1987-94.

54. Requena A, Bronet F, Guillen A, Agudo D, Bou C, Garcia-Velasco JA. The impact of in-vitro maturation of oocytes on aneuploidy rate. Reprod BioMed Online. 2009;18:777-83.

55. Benkhalifa M, Demirol A, Menezo Y, Balashova E, Abduljalil AK, Abbas S, Giakoumakis I, Gurgan T. Natural cycle IVF and oocyte in-vitro maturation in polycystic ovary syndrome: a collaborative prospective study. Reprod BioMed Online. 2009;18:29-36.

56. Vieira RC, Barcelos ID, Ferreira EM, Martins WP, Ferriani RA, Navarro PA. Spindle and chromosome configurations of in vitro-matured oocytes from polycystic ovary syndrome and ovulatory infertile women: a pilot study. J Assist Reprod Genet. 2011;28:15-21.

57. Zhu L, Han CS, Cao ZL, Wang ZB, Han RG, Wang B, Sun QY. Confocal microscopic analysis of the spindle and chromosome configurations of in vitro-matured oocytes from different types of polycystic ovary syndrome patients. Gynecol Obstet Investig. 2015;80:179-86.

58. Zhang XY, Ata B, Son WY, Buckett WM, Tan SL, Ao A. Chromosome abnormality rates in human embryos obtained from in-vitro maturation and IVF treatment cycles. Reprod BioMed Online. 2010;21:552-9.

\section{Publisher's Note}

Springer Nature remains neutral with regard to jurisdictional claims in published maps and institutional affiliations.

Ready to submit your research? Choose BMC and benefit from:

- fast, convenient online submission

- thorough peer review by experienced researchers in your field

- rapid publication on acceptance

- support for research data, including large and complex data types

- gold Open Access which fosters wider collaboration and increased citations

- maximum visibility for your research: over $100 \mathrm{M}$ website views per year

At BMC, research is always in progress.

Learn more biomedcentral.com/submissions 